\title{
La costilla laminar del Instituto de Ciencias de la Construcción Eduardo Torroja (IETcc-CSIC). Levantamiento mediante láser-escáner y evaluación estructural
}

\author{
The riblike sheet of the Eduardo Torroja Institute (IETcc-CSIC). \\ Survey by laser-scanner and structural assessment \\ L. Echevarría $^{(*)}$, C. Garnica ${ }^{(*)}$, J. P. Gutiérrez ${ }^{(*)}$
}

RESUMEN

La capilla exterior construida en el patio de los Alarifes del Instituto de Ciencias de la Construcción Eduardo Torroja fue concebida en forma de «costilla laminar» en 1969 como símbolo de las posibilidades que la tecnología del hormigón armado ofrecía en aquella época. El resultado fue una lámina de hormigón armado de $10 \mathrm{~m}$ de longitud y 6,5 m de altura con espesores que varían entre 6 y $40 \mathrm{~cm}$ y definida a partir de una directriz en forma de Lemniscata de Bernoulli.

Este artículo estudia el comportamiento que ha tenido esta estructura 45 años después de su construcción. Se ha utilizado una herramienta novedosa como el láser-escáner para el levantamiento volumétrico de la estructura y la modelización tridimensional para realizar el cálculo mediante MEF. Se ha hecho una evaluación de los desperfectos observados en la lámina, un estudio de las deformaciones sufridas y un análisis de su comportamiento resistente y seguridad estructural. A partir de la investigación realizada puede concluirse que la construcción puede mantenerse en servicio realizando las reparaciones y el mantenimiento adecuados.

Palabras clave: Lámina de hormigón; seguridad; fluencia; cálculo numérico.

\section{ABSTRACT}

The outer chapel built in the Alarife's courtyard of the Eduardo Torroja Institute was designed as a riblike sheet in 1969 as a symbol of the possibilities that reinforced concrete technology offered at that time. The result was a sheet of reinforced concrete $10 \mathrm{~m}$ long and $6.5 \mathrm{~m}$ high with thicknesses ranging between 6 and $40 \mathrm{~cm}$ and defined from a guideline whose form Lemniscate of Bernoulli.

This article studies the behavior that this structure has had 45 years after its construction. A novel tool such as laserscanner has been used for volumetric survey of the three-dimensional structure to perform the calculation using FEM modeling. An evaluation of the damages observed in the sheet, a study of the strains and an analysis of its resistant behavior and structural safety have been made. From the research conducted it can be concluded that the construction can be kept in service performing repairs and maintenance.

Keywords: Concrete shell; safety; creep; numerical calculation.

(*) Instituto de Ciencias de la Construcción Eduardo Torroja (IETcc-CSIC). Madrid (España). Persona de contacto/Corresponding author: 1.echevarria@csic.es (L. Echevarría)

Cómo citar este artículo/Citation: Echevarría, L., Garnica, C., Gutiérrez, J. P. (2014). La costilla laminar del Instituto de Ciencias de la Construcción Eduardo Torroja (IETcc-CSIC). Levantamiento mediante láser-escáner y evaluación estructural. Informes de la Construcción, 66(536): e038, doi: http://dx.doi.org/10.3989/ic.14.116.

Licencia/License: Salvo indicación contraria, todos los contenidos de la edición electrónica de Informes de la Construcción se distribuyen bajo una licencia de uso y distribución Creative Commons Reconocimiento no Comercial 3.o. España (cc-by-nc). 


\section{INTRODUCCIÓN}

La necesidad de una nueva capilla en el Instituto de Ciencias de la Construcción Eduardo Torroja (IETcc) en 1969 sirvió de motivo para diseñar y construir la singular "costilla laminar" que se alza en el patio de los Alarifes del Centro. La construcción de este elemento estructural pretendía dar continuidad al espíritu creador que Eduardo Torroja había transmitido a sus obras y al Instituto.

El Instituto de Ciencias de la Construcción Eduardo Torroja (IETcc) se aloja desde 1953 en el edificio sito en el barrio de Costillares, próximo a la calle Arturo Soria en el norte de Madrid (1). Esta institución, perteneciente al Consejo Superior de Investigaciones Científicas (CSIC) desde 1946, se dedica a la investigación en el campo de la construcción y sus materiales y surgió de la fusión del Instituto de la Construcción y la Edificación con el Instituto del Cemento en 1949, recibiendo el nombre de Instituto Técnico de la Construcción y el Cemento. Posteriormente, tras la muerte en 1961 de su creador y primer director, Eduardo Torroja Miret, su nombre se incorpora a la denominación oficial del centro como homenaje póstumo a esta destacada figura internacional de la ingeniería y la construcción.

La actual sede del IETcc, concebida por Eduardo Torroja y sus colaboradores, se compone de un edificio central formado por varios cuerpos que se extienden por el solar conformando diversos patios y atractivos espacios arbolados exteriores, ideados para ser los escenarios del esparcimiento y encuentro de trabajadores y visitantes. Esta obra destaca tanto por la interesante organización espacial del conjunto como por las múltiples aportaciones singulares que se realizaron en su creación, convirtiendo el diseño y construcción del edificio en un verdadero taller experimental. Como resultado, se obtuvo un original compendio de innovadores espacios arquitectónicos y diseños estructurales conformando los elementos más singulares de esta sede. Caben destacar entre estos elementos el comedor circular, la cubierta laminar triangulada de talleres y naves, la sustentación mediante cables de la entreplanta del hall de acceso al salón de actos, así como otros detalles estructurales constructivos y arquitectónicos del Centro (2).

Durante las dos primeras décadas de funcionamiento de esta singular sede, la plantilla del IETcc creció considerablemente, de modo que la capilla de que se disponía, construida en torno a 1956, resultaba insuficiente. Se consideró como solución óptima la construcción de una capilla exterior en torno a la cual pudieran concentrarse todos los miembros del Instituto, aprovechando de esta forma los espacios exteriores del complejo concebidos desde un principio como espacios singulares.

El lugar elegido para situar la estructura fue el denominado patio de los Alarifes, espacio ajardinado que se encuentra delimitado por el ala de talleres, la nave de hormigones y la nueva nave de ensayos, que fue inaugurada un año antes de la construcción de la lámina. El patio contenía una escultura consistente en un atractivo juego espacial con varios perfiles laminados de acero que representaba la importancia que tenían estos elementos en la construcción y, por lo tanto, en las actividades del Instituto.

El diseño de esta nueva obra coincidió con la proximidad de la celebración en Madrid del Congreso Internacional de la Asociación Internacional de Estructuras Laminares en honor a Eduardo Torroja, quien fue su primer presidente y fundador. Este hecho animó a proyectar una estructura laminar que actuase a la vez de nuevo símbolo en Costillares y de capilla para las festividades y celebraciones señaladas. Por ello, se escogió la geometría de costilla que ya se encontraba presente en algunas zonas del recinto y que tenía clara relación con el nombre de la zona donde se encuentra la institución, el barrio de Costillares, optando así por construir una costilla laminar (3), Figura 1.

Desde un principio se consideró que la capilla debía ser lo más sencilla posible en relación a sus elementos constructivos, de modo que una vez definida la geometría estructural, esta misma actuaría de contenedor arquitectónico acogiendo el programa funcional especificado. Pretendiendo de esta forma que los aspectos formal y estructural quedasen ligados, el uso de hormigón armado como material de construcción parecía la opción idónea. La geometría de costilla laminar en hormigón armado permitía conjugar en un solo elemento la estructura portante y la cubierta del espacio sagrado, cumpliendo eficazmente ambas funciones mediante una hoja continua de este material.

La presencia del monumento a los perfiles laminados en el patio elegido para la obra motivó la idea de construir el nuevo elemento de hormigón adherido al mismo y añadir un pe-
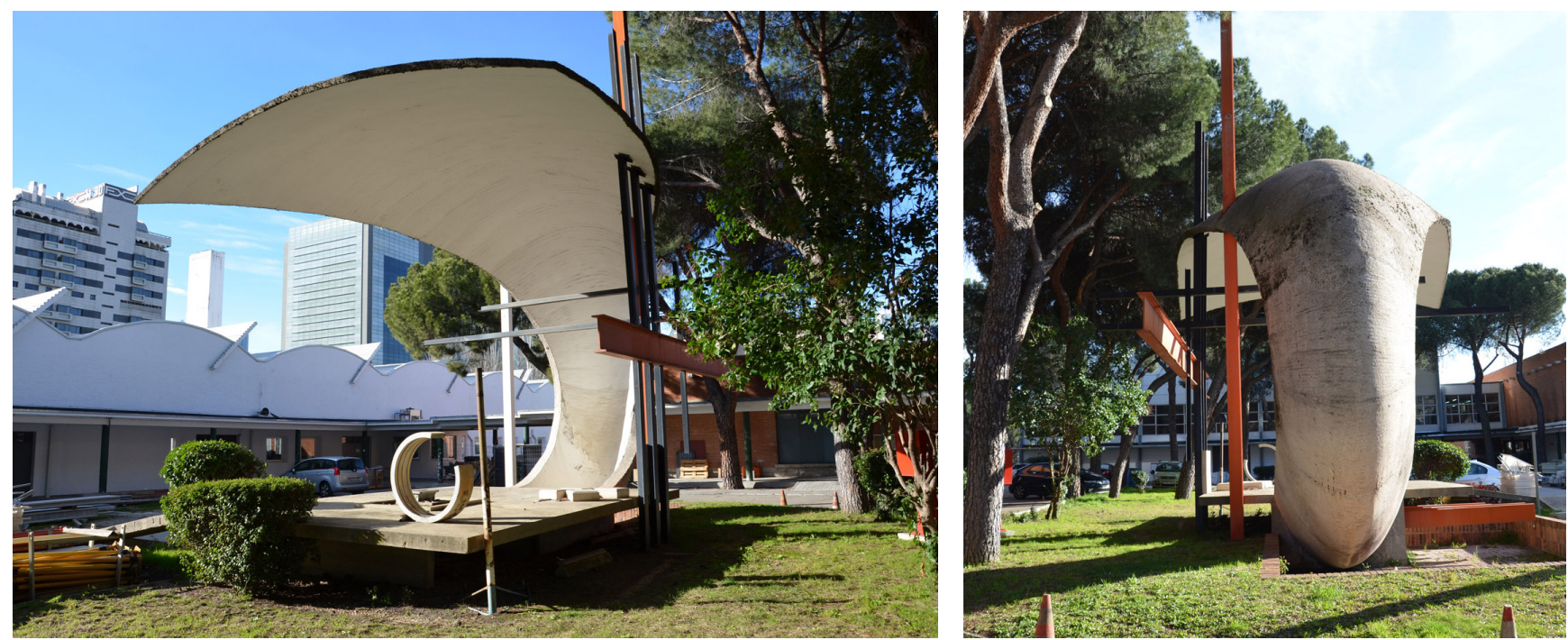

Figura 1. Fotografías de la capilla, año 2013 (autoría: Ma Rosa Gómez del Álamo). 

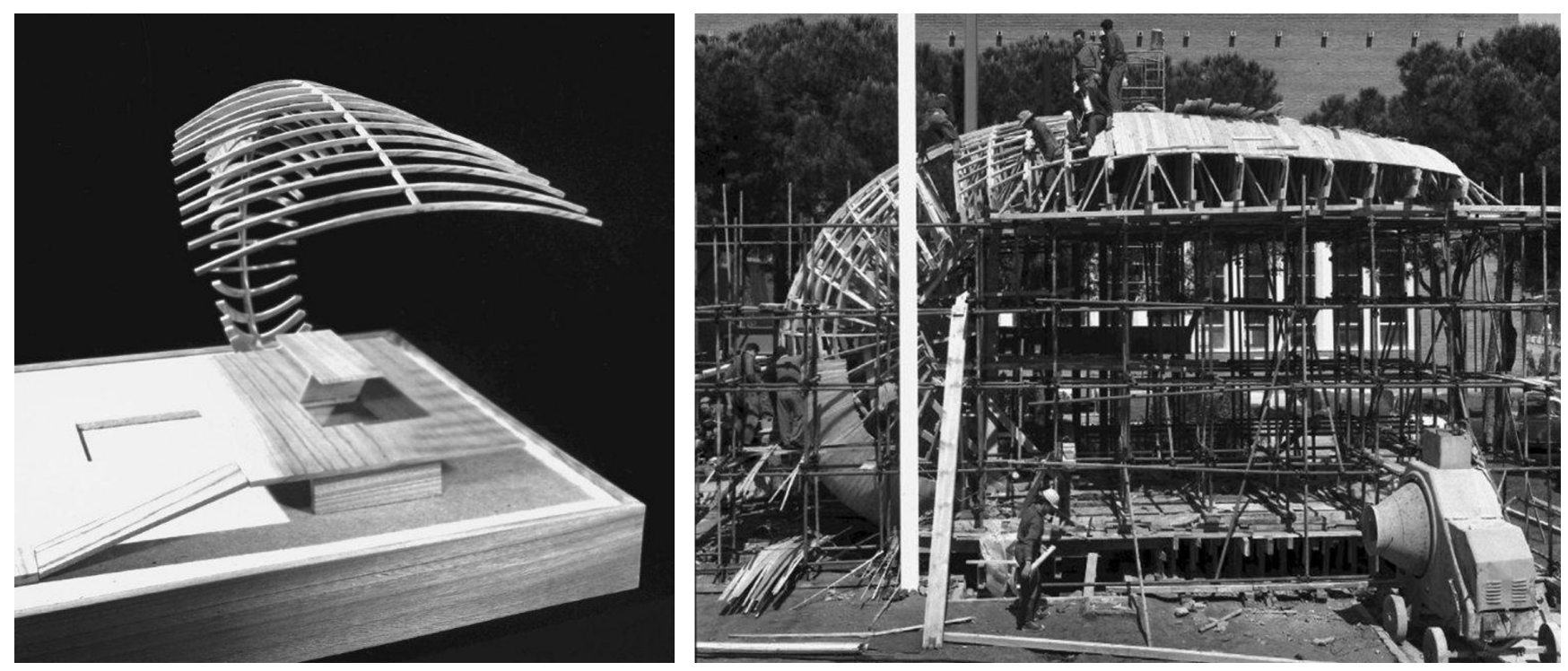

Figura 2. Fotografías de la maqueta de concepción de la capilla y de su construcción (Archivo del IETcc).

queño estanque de envoltura cerámica en la parte trasera, de modo que el conjunto completaba la antigua escultura de acero para erigirse en la nueva capilla que sería a la vez símbolo de los materiales más importantes de construcción en aquella época: acero, hormigón y cerámica.

En el proyecto y construcción intervinieron varios ingenieros y arquitectos del Instituto. La construcción de la capilla, Figura 2, se realizó en un plazo de tiempo considerablemente corto para este tipo de obras debido a la necesidad que había de inaugurar el espacio religioso en una fecha concreta que coincidía con una determinada celebración. Por ello se utilizó un hormigón con cemento blanco de endurecimiento rápido que permitió desencofrar a los 3 días de su colocación en obra. Se terminó en 15 días, un plazo muy ajustado teniendo en cuenta la complejidad de los encofrados basados en cimbras y de la geometría de las armaduras. El hormigón utilizado se dosificó con el objetivo de obtener una resistencia de $20 \mathrm{MPa}$ a los tres días. Las probetas ensayadas a esta edad presentaron una resistencia de $19 \mathrm{MPa}$. El acero utilizado para armar la lámina se dispuso en barras de diámetro comprendido entre 12 y 20 mm, con un límite elástico de $400 \mathrm{MPa}$.

\section{GEOMETRÍA DE PROYECTO}

En la documentación existente en los archivos del IETcc y en publicaciones de la época se encuentra definida la geometría de la costilla laminar que da forma al elemento principal de esta capilla. A partir de ella se han construido modelos informáticos tridimensionales que permiten estudiar esta lámina de doble curvatura en toda su complejidad formal.

La geometría de esta lámina se estableció en proyecto definiendo matemáticamente, en primer lugar, las curvas que forman su intradós y a partir de estas se aplicaron los espesores que se calcularon necesarios para dotar a cada zona de la capilla de las propiedades mecánicas necesarias para cumplir su función estructural. La superficie que conforma el intradós de esta lámina queda definida por una curva que actúa de directriz y eje de simetría y por las curvas perpendiculares a ella que, a modo de generatrices, constituyen las secciones radiales de esta superficie manteniendo la continuidad de su curvatura en toda su extensión.
La directriz del intradós está inspirada en las formas de costilla que se encuentran presentes en diferentes zonas del Instituto y está compuesta por un fragmento de lemniscata de Bernoulli que responde a la ecuación $\rho=c \cdot \sqrt{\sin 2 \alpha}$ (donde $c$ es el máximo radio, que toma el valor de 8,9 metros); y por un arco de circunferencia de $24 \mathrm{~m}$ de radio que continúa tangencialmente desde el extremo en voladizo de la lemniscata. A partir de esta directriz se desarrolla la superficie mediante parábolas de segundo grado a modo de generatrices, posicionando el punto medio de las mismas sobre dicha directriz. Las generatrices parabólicas van modificando su geometría a lo largo de la directriz en base a varias funciones matemáticas que aseguran la variación de la curvatura transversal de la superficie manteniendo la continuidad de su curvatura longitudinal, Figura 3.

Una vez definido el intradós de este elemento singular, se definió su extradós, dotando a la lámina de diferentes espesores en función de las secciones que estructuralmente se necesitaban en cada zona, así como de la esbeltez y estética que se pretendía mostrar. Estas variaciones de espesores responden también a un conjunto de leyes analíticas que aseguran la continuidad del extradós de la lámina y de sus bordes, así como de su curvatura. En el eje central de la estructura, defi-

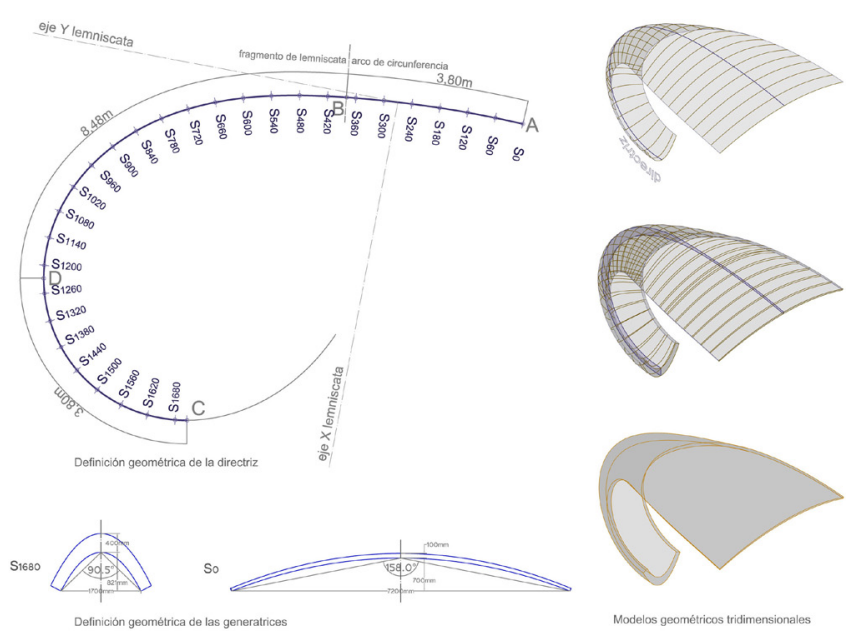

Figura 3. Estudio geométrico de la costilla laminar. 


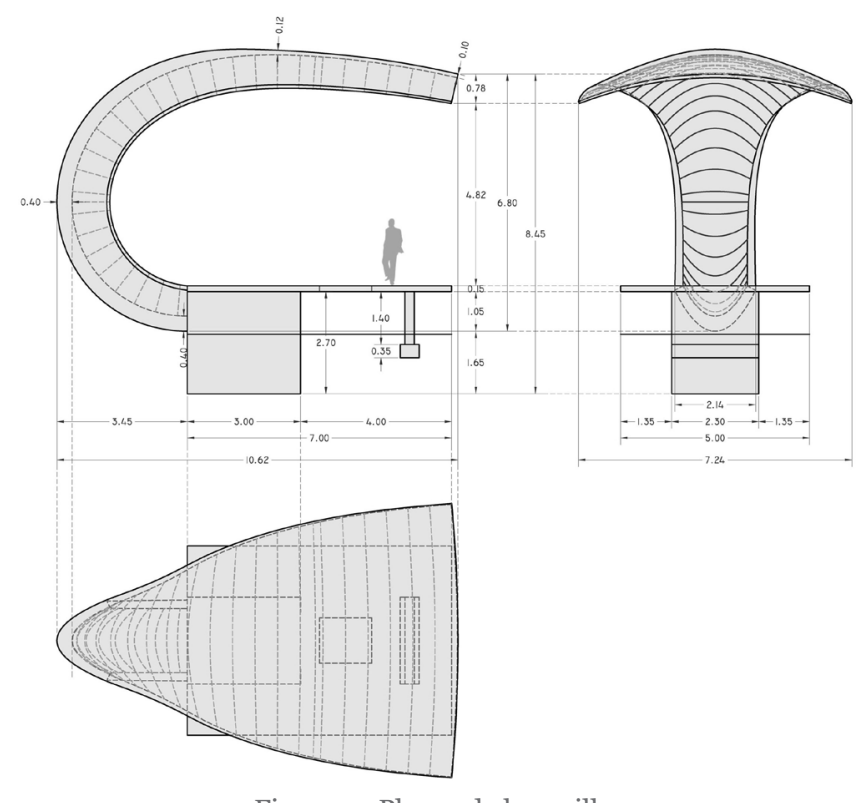

Figura 4. Planos de la capilla.

nido por la directriz, los espesores varían desde $40 \mathrm{~cm}$ en el tramo de arranque en cimentación hasta $10 \mathrm{~cm}$ en el extremo en voladizo. En los bordes laterales de la lámina los espesores quedan comprendidos entre $25 \mathrm{~cm}$ en el arranque inferior y $6 \mathrm{~cm}$ en el extremo en ménsula. Estas leyes de espesores confieren a esta costilla laminar las propiedades mecánicas necesarias para soportar las solicitaciones a que se ve sometida en cada tramo, siendo más severas en las zonas inferiores, y proporciona al elemento de hormigón unas estilizadas proporciones en sus bordes que dotan a la capilla de un atractivo aspecto de ligereza.

La lámina resultante presenta una longitud en planta superior a los $10 \mathrm{~m}$, con $17 \mathrm{~m}$ de recorrido curvo, más de $7 \mathrm{~m}$ de anchura en planta y una altura de 6,5 m, Figura 4 .

\section{LEVANTAMIENTO}

El análisis de esta estructura singular requería obtener datos precisos sobre su estado actual, pero, debido a su complejidad geométrica, realizar un levantamiento suficientemente deta- llado se presentaba como un problema a resolver. Se probaron diferentes métodos de medición topográfica como son el uso de estación total y la fotogrametría, valorando su complejidad y la precisión de los datos obtenidos con ellos. Finalmente, la posibilidad de utilizar sistemas de medición láser se convirtió en el método más práctico para este tipo de elementos por sus características de precisión y comodidad. El levantamiento mediante láser escáner se realizó en colaboración con la empresa Grafinta, utilizando un equipo compacto Z+F IMAGER 5010 cuyas principales características técnicas son: tomas de $320^{\circ}$ verticales y $360^{\circ}$ horizontales; alcance: $0,3-187,3 \mathrm{~m}$; resolución: $0,1 \mathrm{~mm}$; error lineal < $1 \mathrm{~mm}$; resolución máxima: 100000 píxeles; toma de fotografías HDR, Figura 5.

Estos sistemas de medición se basan en la proyección de miles de rayos láser hacia el entorno que rodea al equipo obteniendo datos de la distancia y posición a la que se encuentran los objetos a su alrededor. Con esta información se genera un modelo tridimensional con las posiciones de todos estos puntos obtenidos, a los que se les añade color mediante las fotografías HDR que el mismo equipo realiza durante el escaneo. De cada toma que el equipo realiza se obtiene un modelo tridimensional de puntos que presenta vacíos de información debido a que los propios objetos del entorno actúan de obstáculo a los rayos y por lo tanto no permiten medir los objetos que se encuentran detrás de ellos. Por esta razón, suele ser necesario realizar varias tomas alrededor de la geometría que se pretende levantar para conseguir un modelo completo de la misma, Figura 6.

En este caso el proceso de levantamiento en sí mismo y su idoneidad para el trabajo que se estaba realizando era parte de la investigación. Por ello se realizaron múltiples tomas desde diferentes puntos rodeando la estructura sin necesidad de ser austeros con los recursos utilizados. En dos mañanas se realizaron un total de 17 tomas que posteriormente se procesaron con el software específico para obtener las 17 nubes de puntos correspondientes a partir de las cuales se generaría el modelo tridimensional de trabajo. La cantidad de información que contiene cada una de estas nubes escaneada a alta resolución es considerable y el manejo de las mismas requiere ordenadores y programas de altas prestaciones.

Con las 17 nubes de puntos creadas, el siguiente paso consistió en combinarlas todas en un único modelo. Para ello exis-
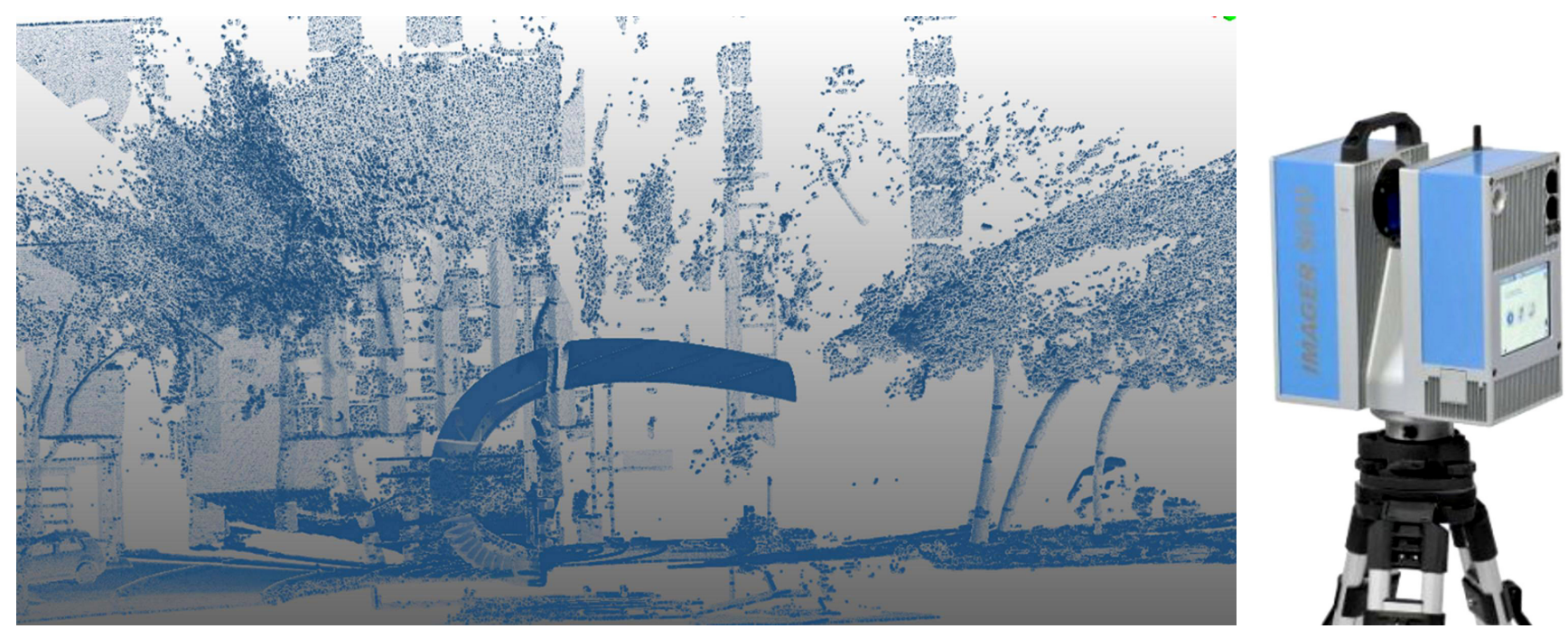

Figura 5. Nube de puntos isocroma y equipo Z+F IMAGER 5010. 

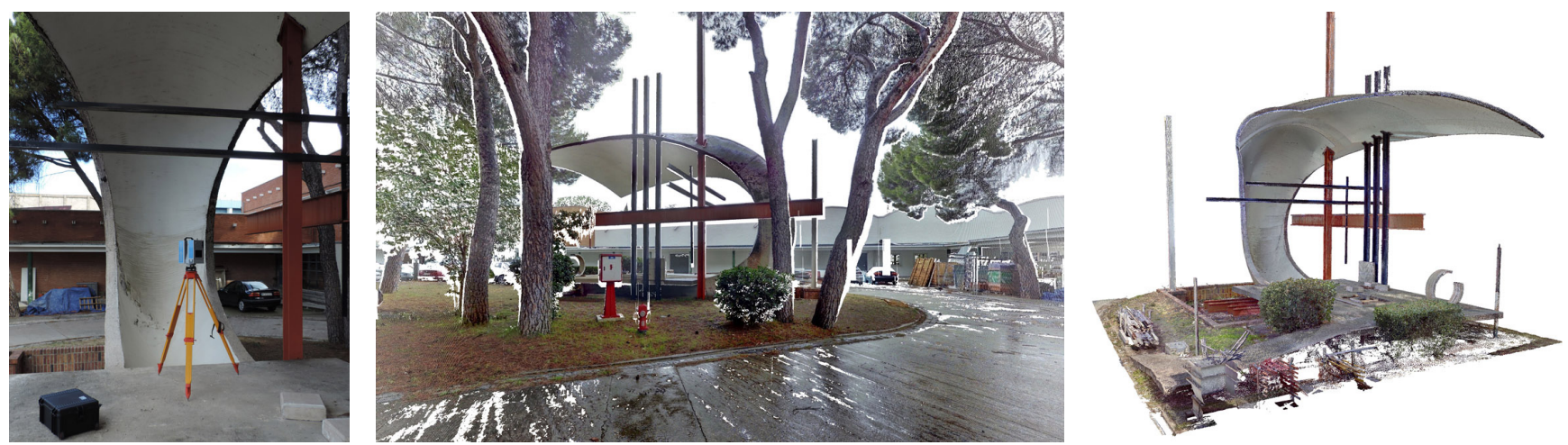

Figura 6. Fotografía del proceso de levantamiento y nubes de puntos RGB obtenidas.

ten diversos métodos que permiten relacionar los sistemas de coordenadas de cada nube con el objetivo de mantener la precisión topográfica deseada al fusionarlas. Algunos métodos se basan en el uso de dianas físicas colocadas junto al modelo durante las tomas, de modo que el programa que acopla las nubes tenga unas referencias comunes en todas ellas. En este caso de estudio se ha utilizado métodos informáticos basados en análisis geométricos que permiten unificar las nubes de manera automática buscando coincidencias geométricas en todas las nubes de puntos. El resultado del levantamiento es un modelo tridimensional con millones de puntos de color que muestra el objeto escaneado con gran realismo y precisión, Figura 7.

En el trabajo que se trata en este artículo se necesitaba comparar el modelo tridimensional construido a partir de la documentación obtenida del proyecto original con el modelo obtenido del levantamiento. Con este fin fue necesario convertir el modelo de nubes de puntos, resultado de los escaneos láser, en mallas tridimensionales mediante software especializado y se extrajo la superficie que conforma el intradós de la costilla laminar para compararla con su equivalente del modelo geométrico de proyecto. Los resultados de esta comparación muestran las deformaciones sufridas por la estructura con el paso de los años.

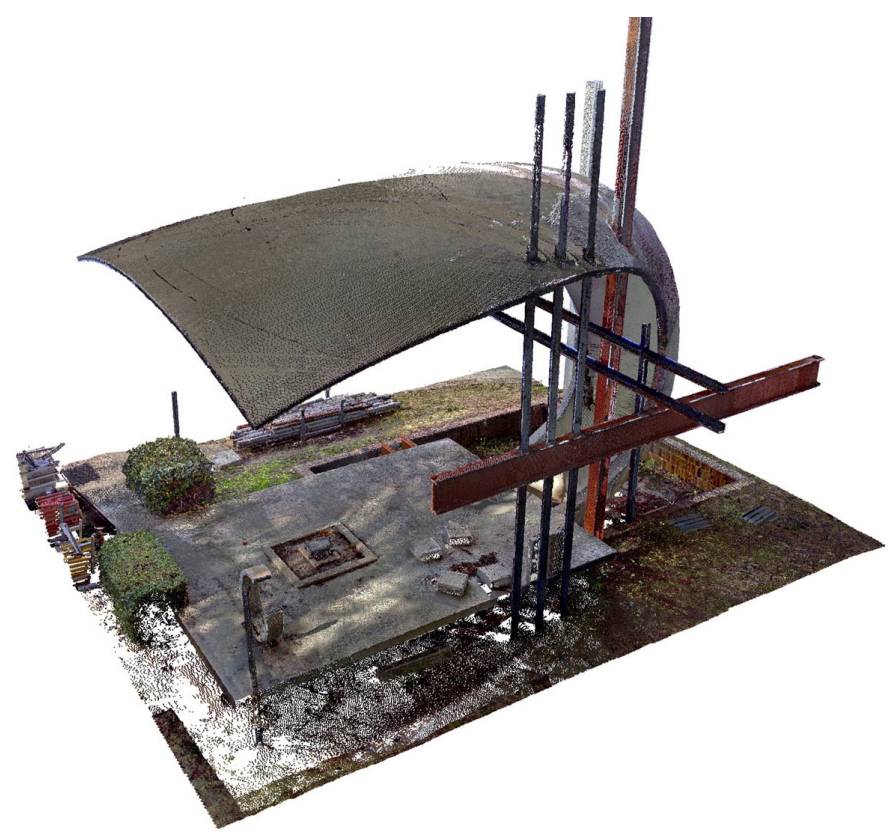

\section{DEFORMACIONES}

El levantamiento mediante técnicas laser que se ha explicado en el apartado anterior ha permitido obtener un modelo suficientemente preciso de la superficie que da forma al intradós de la lámina con el objetivo de analizar las deformaciones que ha sufrido esta estructura desde que fue construida. Para estudiar estos procesos se ha comparado esta superficie con su equivalente del modelo construido a partir de la documentación disponible del proyecto de la capilla. Ambas superficies se han acoplado en un mismo modelo usando como referencia común el arranque de las mismas desde la cimentación, con la finalidad de evitar que los posibles asientos y giros de la cimentación interfieran en este análisis.

Seccionando las superficies con un plano vertical por sus ejes de simetría se obtiene la directriz original de proyecto y la deformada de ésta tras 45 años de vida de la lámina. En la Figura 8 se muestran estas secciones cuantificando los desplazamientos radiales que se han producido en este eje de la costilla laminar. La deformada de la directriz, en color rojo en esta figura, muestra un claro descenso del extremo en voladizo que lleva asociado el desplazamiento hacia el exterior del tramo vertical de la curva. Los signos positivos de la figura indican desplazamientos hacia el interior de la directriz y los signos

Figura 7. Nube de puntos RGB y comparación del modelo teórico con el modelo mallado obtenido con el equipo laser. 


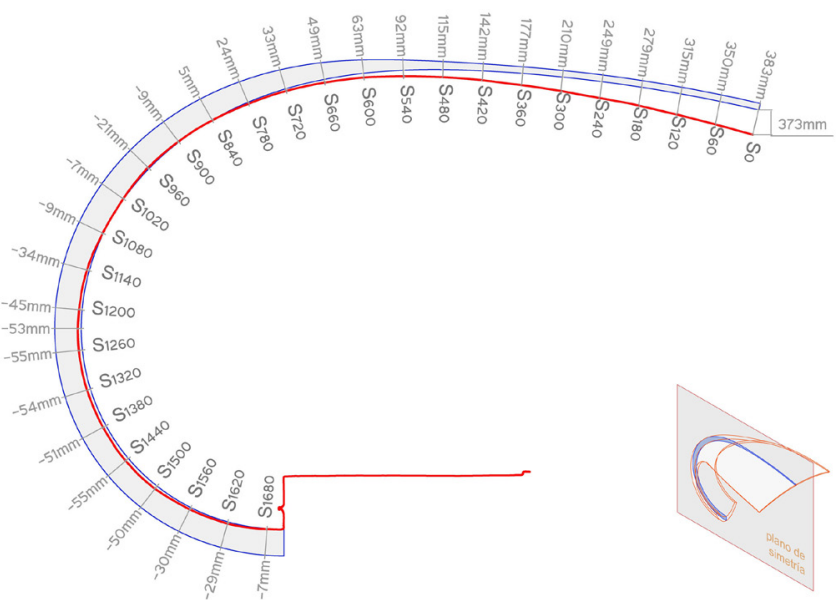

Figura 8. Deformación actual en la sección por el plano de simetría.

negativos hacia el exterior. Las cargas permanentes a que se ve sometida la lámina son las cargas de peso propio, que son las responsables de este marcado descenso del extremo en ménsula, del orden de $373 \mathrm{~mm}$, debido al fenómeno de fluencia que ha sufrido el hormigón por haber estado sometido a estas cargas durante más de cuatro décadas, y del desplazamiento horizontal de unos $55 \mathrm{~mm}$ que ha sufrido el tramo vertical.

Los datos disponibles sobre la construcción de la obra (3) indican un descenso de $120 \mathrm{~mm}$ en el extremo del voladizo de la capilla tras el desencofrado a los 3 días. Esto significaría que las deformaciones debidas a la fluencia del hormigón podrían cuantificarse en el doble de la deformación instantánea inicial, valores que se corresponden con los indicados en las normativas y publicaciones técnicas de aquella época para este tipo de fenómenos.

Para el análisis de las deformaciones transversales se han seccionado las superficies mediante los planos perpendiculares a la directriz que contienen las generatrices parabólicas que dan forma al intradós de la capilla. En la Figura 9 se recogen algunas de estas secciones que representan los tramos más significativos de la lámina comparando las generatrices de la geometría de proyecto con sus respectivas deformadas. Se puede observar que se produce un fenómeno de apertura de las secciones parabólicas que está asociado al descenso de la ménsula que se ha explicado anteriormente. Estas aperturas de las generatrices son bastante acusadas en los tramos centrales de la costilla laminar, siendo mucho menores en los extremos y especialmente en el arranque desde la cimentación debido a la coacción que éste supone.

Las deformaciones estudiadas se han producido de manera bastante simétrica respecto del plano de simetría de la estructura y pueden resumirse en un importante descenso del extremo en voladizo de la lámina de hormigón que conlleva un desplazamiento horizontal hacia el exterior del tramo vertical de la misma en el plano de simetría y la apertura transversal de las generatrices a lo largo de prácticamente toda la lámina.

\section{INSPECCIÓN DE LA ESTRUCTURA}

La estructura que se está evaluando es una lámina de hormigón armado de pequeño espesor (entre 6 y $40 \mathrm{~cm}$ ), en el estado actual con el hormigón cara vista en la parte exterior de la lámina y una capa de pintura, de color blanco, en el in- tradós. Estas características de la lámina, unidas al hecho de que la lámina ha estado a la intemperie durante los últimos 45 años, hace que se pueda asegurar que el mayor riesgo para la durabilidad de esta estructura es el ataque del agua (lluvia o nieve) y los ciclos de humedad-sequedad, que pueden haber afectado a algunas armaduras, fundamentalmente allí donde el recubrimiento ha sido insuficiente, a la vez que la carbonatación ha disminuido la protección de las armaduras.

El recubrimiento del armado especificado en proyecto es de $4 \mathrm{~cm}$, sin embargo, la dificultad de construcción de la lámina y el hecho de que no se pudiera ferrallar en taller, pudo provocar que en ciertos puntos este recubrimiento fuera sensiblemente menor. Este efecto se ha producido especialmente en la sección de máxima curvatura, la sección horizontal de la lámina (S1230). Durante la inspección se detectó la presencia de algunas coqueras que dejaron a la intemperie alguna de las armaduras. En estas zonas se ha detectado óxido alrededor de las barras de acero, Figura 10. No se han detectado fisuras en las superficies ni disgregación o meteorización del material en la misma, ni ningún otro síntoma que pudiera significar un comportamiento anómalo de la estructura, aunque en diversas zonas de la superficie exterior se ha detectado suciedad. Se puede concluir que la oxidación y los desperfectos manifestados son localizados y de poca entidad, por lo que se puede afirmar que la integridad de la lámina está asegurada.

\section{EVALUACIÓN ESTRUCTURAL}

\subsection{Normativa de proyecto y construcción de la costilla laminar}

La evaluación estructural de la lámina comienza con un estudio de la normativa que se encontraba vigente en el momento de diseño y construcción de la estructura, la «Instrucción para el proyecto y la ejecución de obras de hormigón en masa o armado» de 1968 (4), que derogaba la Instrucción para el Proyecto de Obras de Hormigón de 1944, que a su vez consistía en una revisión de la norma de 1939. Una disposición transitoria permitía que, durante los dos años siguientes a la aprobación de la EH-68, fuera posible emplear cualquiera de estas dos normas, la EH-1944 o la EH-1968.
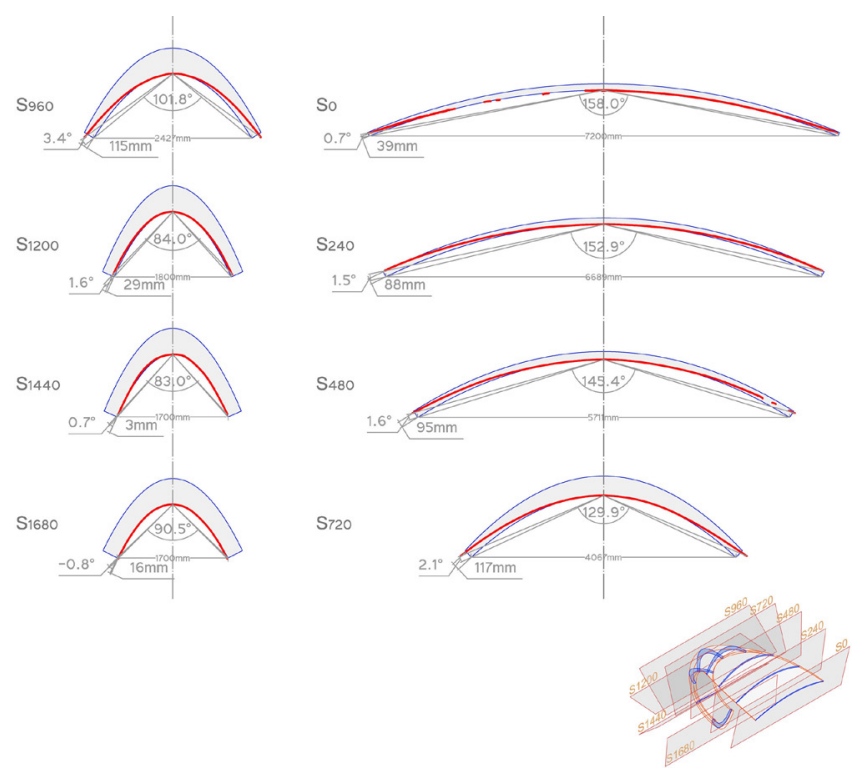

Figura 9. Deformaciones de las secciones transversales radiales. 

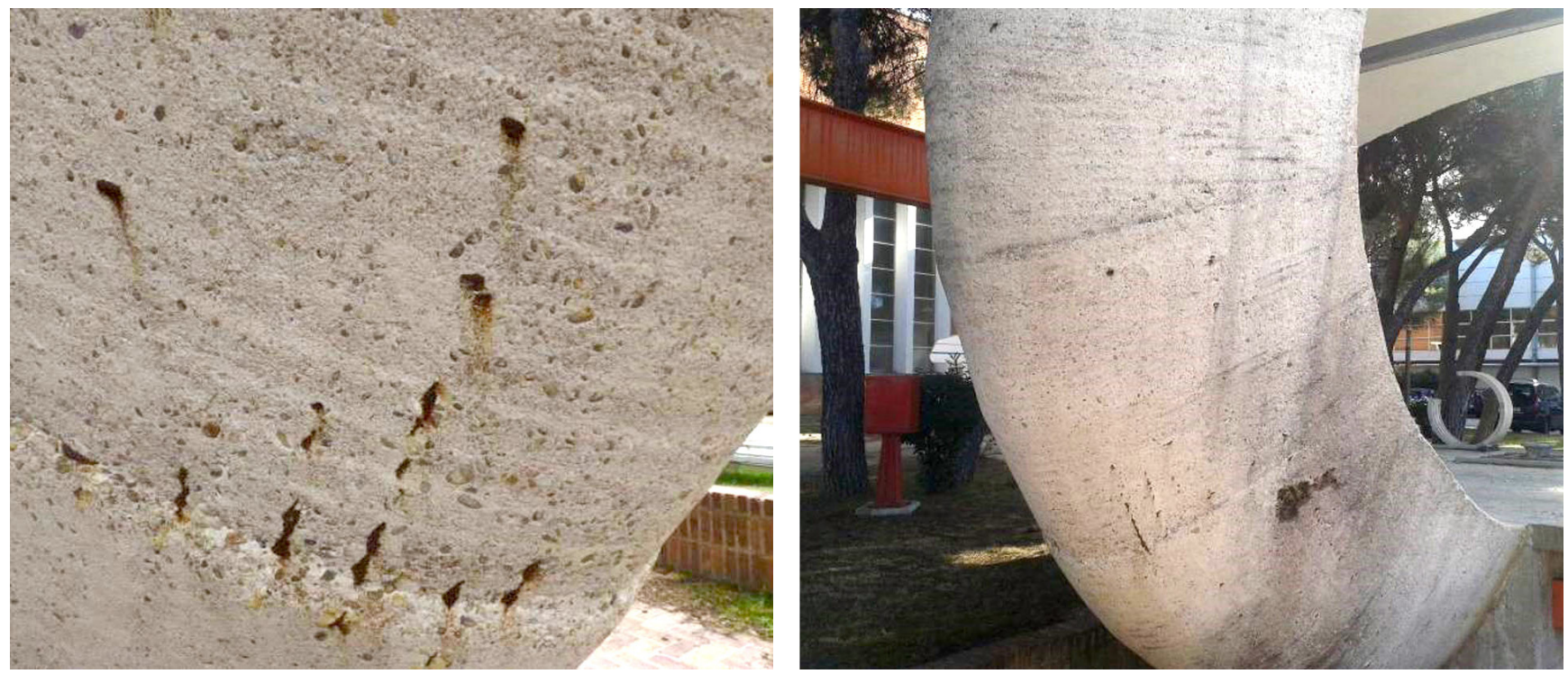

Figura 10. Oxidación localizada de la lámina.

\section{Materiales}

La Instrucción EH-68 distingue entre dos tipos de barras de acero, las barras de acero ordinario y las barras de acero de alta adherencia (aquellas con límite elástico superior a $360 \mathrm{MPa}$, alargamiento a rotura superior al $1 \%$ y que superen un ensayo de arrancamiento).

En cuanto al hormigón, la EH-68 establecía que para obras de hormigón armado su resistencia debía ser mayor de 12 MPa y en este caso la densidad a emplear en el cálculo de la estructura debía ser de $2,4 \mathrm{t} / \mathrm{m}^{3}$.

\section{Introducción de la seguridad}

La EH-68 ya incluía una serie de coeficientes de minoración que se aplicaban a la resistencia de los materiales y de mayoración a las acciones para dimensionar y verificar la estructura.

La resistencia de cálculo del acero a tracción se establecía como el mínimo del límite elástico, reduciéndolo con un coeficiente de minoración de 1,1 y del límite de rotura reducido con un coeficiente de 1,3. La resistencia del acero a compresión es igual a la de tracción pero limitada a $400 \mathrm{MPa}$. En cuanto al hormigón, se establecía la resistencia de cálculo como la resistencia característica minorada por un coeficiente de 1,5 . Las acciones se mayoraban, en el caso general, aplicando un coeficiente de 1,5, pudiendo este valor disminuirse hasta 1,35 bajo justificación previa.

\section{Método de cálculo}

El método general de rotura que se recogía en la EH-68 frente a solicitaciones de flexocompresión era el del momento tope, aunque se permitía la utilización de otros diagramas tensióndeformación experimentales como el parábola-rectángulo. Para calcular la resistencia a cortante, la norma ya consideraba por separado la contribución del hormigón y de las armaduras transversales.

El cálculo de la lámina se realizó determinando los esfuerzos principales en el plano vertical de simetría como si fuera una ménsula o voladizo. Los esfuerzos seccionales que se produ- cen se determinan de forma aproximada teniendo en cuenta las distintas zonas en el desarrollo de la lámina, consistente básicamente en 3 ó 4 zonas en función de la curvatura de las secciones transversales de la lámina, como se indica en (3).

\subsection{Recálculo estructural}

Para poder realizar el cálculo de la estructura se ha aprovechado el modelo 3D obtenido a partir de la definición teórica de la estructura. El proceso seguido para la realización del recálculo de la lámina se ha basado en dos partes consecutivas. En primer lugar, se ha introducido la lámina en diversos programas de elementos finitos, obteniendo así las tensiones y deformaciones que debe soportar la estructura para, a continuación, comprobar, mediante un programa realizado en el IETcc, las secciones más representativas de la lámina.

Las acciones principales que se han introducido a la hora de comprobar el comportamiento de la lámina han sido el peso propio y una sobrecarga de nieve. Se ha optado por no introducir ninguna sobrecarga debido a la acción del viento, ya que no es determinante a la hora de comprobar la estructura.

Para las acciones de peso propio, se ha considerado una densidad del hormigón de $2,4 \mathrm{t} / \mathrm{m}^{3}$, siguiendo la instrucción del año 1968, que en este caso se ajusta mejor al hormigón y armado empleados.

En cuanto a la sobrecarga de nieve (Figura 11), se decide introducir la carga básica que define la normativa actual de $1 \mathrm{kN} / \mathrm{m}^{2}$, aplicada uniformemente sobre la proyección horizontal de la lámina. Para tratar de evaluar la rigidez lateral de la lámina, se ha estudiado también un caso de carga de nieve aplicado de forma disimétrica sobre una mitad de esta proyección.

Posteriormente, se han calculado las deformaciones que producen en la lámina los efectos de la temperatura. Estas deformaciones son pequeñas, del orden de $2 \mathrm{~mm}$ para una variación de temperatura de $20^{\circ} \mathrm{C}$.

Para el cálculo de las características del hormigón de la lámina se ha empleado la información de los ensayos realizados 


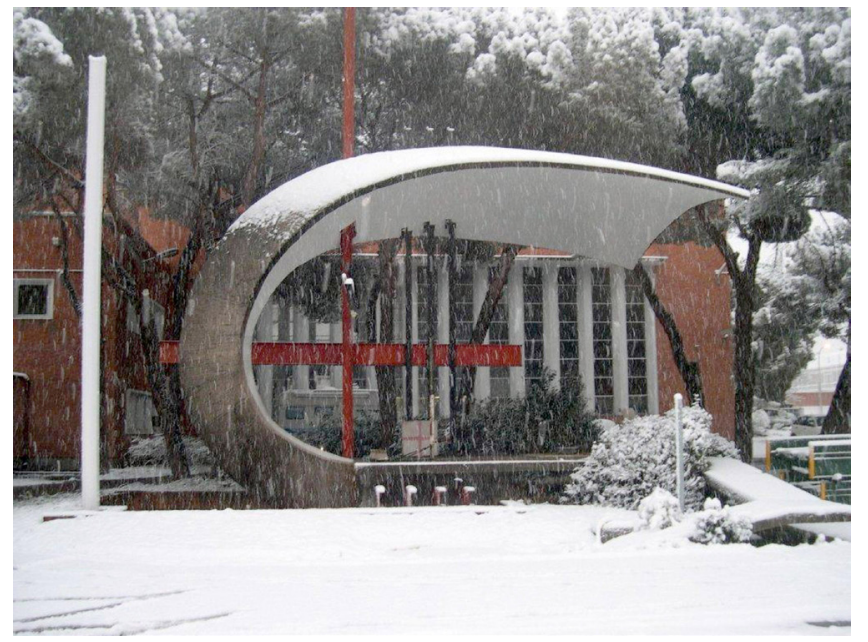

Figura 11. Lámina con carga de nieve.

durante su construcción, que indicaban una resistencia del hormigón a los 3 días de $19 \mathrm{MPa}$ (3). A partir de esta información se ha estimado su módulo de elasticidad empleando las fórmulas recogidas en (5) y (6). De esta forma se puede estimar que la resistencia a 28 días de este hormigón puede estar comprendida entre 30 y $40 \mathrm{MPa}$. Se ha optado por emplear en los cálculos un valor asumible de $35 \mathrm{MPa}$. Aplicando estas fórmulas, se obtiene un módulo de elasticidad entre 22,6 GPa y 26,7 GPa a los 3 días, según se considere un módulo secante o un módulo tangente. Una estimación del módulo de elasticidad equivalente teniendo en cuenta la fluencia, estimada según las directrices de la EHE-08, proporciona un valor de 7,7 GPa. El acero empleado en la estructura tiene un límite elástico de $400 \mathrm{MPa}$.

\section{Modelos numéricos}

Debido a la complejidad geométrica que presenta la estructura se han realizado dos modelos de cálculo por ordenador de la estructura.

En un primer momento se realizó un sencillo modelo tridimensional de barras con el objetivo de obtener, de manera aproximada, una serie de datos (tensiones, flechas, etc.) para calibrar los demás modelos de cálculo.

El segundo modelo de cálculo se realizó mediante elementos finitos. Para poder realizar este modelo fue necesario, en un primer lugar, obtener una geometría teórica a partir de la información obtenida del proyecto de la estructura, para a continuación poder introducirla en el programa de elementos finitos. Una vez definida la geometría de la lámina, se empleó una malla densa de elementos finitos de 20 nodos para su cálculo.

A partir de estos dos modelos se han obtenido como resultados las tensiones y desplazamientos de la lámina.

En la Figura 12 se representa la lámina con el desplazamiento vertical en cada punto. La flecha máxima que se produce es de $223 \mathrm{~mm}$ en el extremo de la lámina para el caso de no linealidad geométrica de la estructura y considerando los efectos de fluencia.

La lámina con las tensiones $\sigma_{z z}$ en cada punto se incluye también en la Figura 12. Como se puede ver, en la sección horizontal en el tramo vertical de la lámina se producen las mayo- res tracciones y compresiones de la estructura. Las tensiones máximas que se producen son de 8,5 MPa de compresión y de 3,6 MPa de tracción.

Al obtener el diagrama de la sección horizontal de la estructura, se puede observar como en esta sección, se produce una distribución típica de tensiones debidas a solicitaciones de flexocompresión, con tracciones y compresiones más elevadas cuanto más nos alejamos de la fibra neutra de la sección, Figura 12.

Por último, a partir de diversas secciones de las que se habían obtenido sus dimensiones y armado, se han calculado los coeficientes de seguridad frente a las acciones previstas (peso propio, y peso propio más nieve) aplicando los criterios de la actual Instrucción EHE-08. Para las secciones más significativas se han obtenido los diagramas de interacción axil momento (7).

La sección horizontal de la lámina situada a la cota 3,42 m es la sección de máximo momento. Para todas las combinaciones de las cargas de peso propio y nieve, las solicitaciones están dentro del diagrama de interacción, encontrándose coeficientes adicionales de seguridad mayores que la unidad. Se ha comprobado esta sección a flexión esviada en la hipótesis de nieve disimétrica actuando en la mitad de la lámina, encontrándose también valores del coeficiente adicional de seguridad frente a las acciones mayor que la unidad. Igualmente, la comprobación de la sección crítica frente a esfuerzo cortante ha resultado con un coeficiente adicional de seguridad superior a la unidad.

Los resultados obtenidos del cálculo de comprobación de las secciones muestran como éstas cumplen sobradamente con los criterios de seguridad que recoge la normativa actual, siendo los métodos de cálculo empleados en aquellos años una buena aproximación de los métodos actuales.

Los cálculos mediante el Método de Elementos Finitos han proporcionado una flecha elástica en la clave del borde libre de $75 \mathrm{~mm}$, mientras que, según se deduce de la documentación existente, en el momento de desencofrar se midió una flecha de $120 \mathrm{~mm}$. Esto significa, por una parte, que el módulo de elasticidad a 3 días del hormigón colocado en obra, pudiera ser menor que el estimado, debido probablemente a los cementos de la época, y por otra, a la influencia de la microfisuración de la lámina que reduce la inercia de las secciones, aumentado en consecuencia las flechas.

También se ha realizado el cálculo de la flecha diferida para el peso propio de la lámina. En este caso, la flecha aumenta hasta los $216 \mathrm{~mm}$ en el extremo del voladizo. Esta flecha alcanza los $223 \mathrm{~mm}$ cuando se calcula teniendo en cuenta la no-linealidad geométrica. Por último se ha obtenido la flecha para la carga de nieve, resultando ser de $25 \mathrm{~mm}$. Estas flechas no tienen en cuenta la fisuración de la lámina de hormigón. La flecha real medida, diferencia entre la geometría original de construcción y la geometría actual levantada es de $373 \mathrm{~mm}$. La diferencia de estas dos medidas puede ser debida a errores de replanteo, a la microfisuración de la lámina así como a la incertidumbre en la estimación de las características del hormigón, especialmente en la determinación del módulo de elasticidad.

Se ha observado un comportamiento de la lámina muy simétrico en las deformaciones con el tiempo, con desviaciones de 


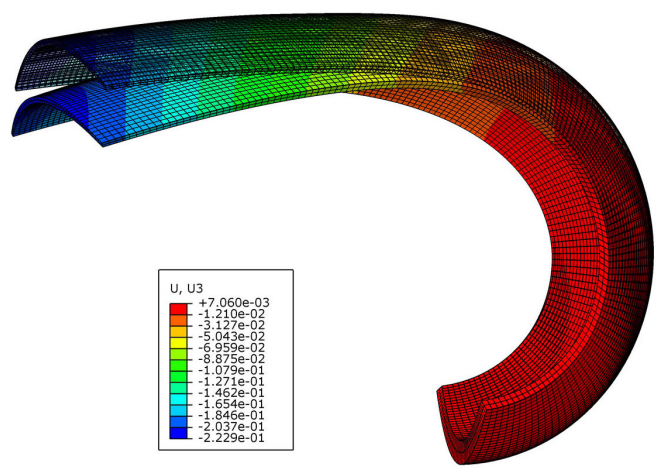

Desplazamientos verticales, factor de escala 4

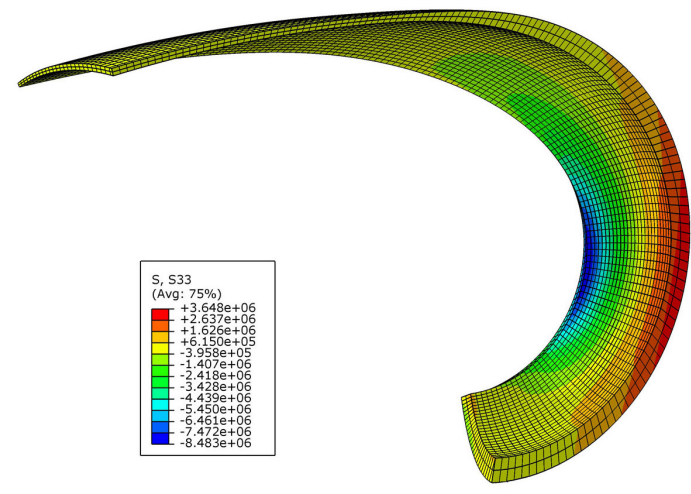

Tensiones $\sigma_{z z}$ en la lámina

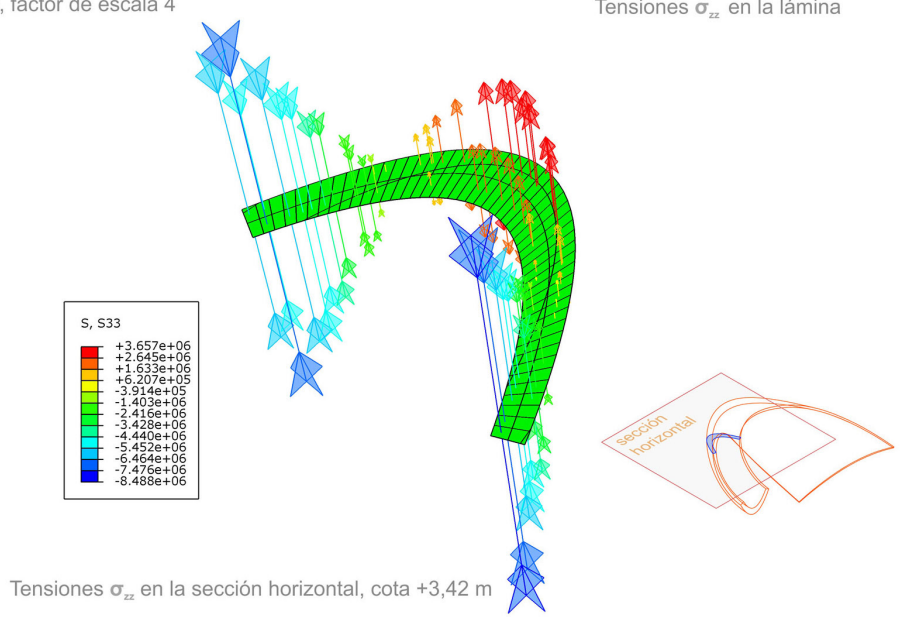

Figura 12. Modelos tridimensionales de cálculo. Desplazamiento vertical con factor de escala 4; tensiones $\sigma_{z z}$ en la lámina y sección horizontal a cota 3,42 m.

escasos milímetros que bien pudieran ser también errores en el replanteo del encofrado de la lámina.

\section{CONCLUSIONES}

El comportamiento de la lámina construida en 1969 ha sido satisfactorio a lo largo de los 45 años de existencia. Las deformaciones más importantes que ha sufrido son debidas a la fluencia del hormigón, ya que los valores del cálculo coinciden con los expresados en las normativas y han resultado ser del orden de 2 veces las deformaciones instantáneas.

Los nuevos métodos de captación de geometría empleados en el levantamiento de la lámina son determinantes cuando las estructuras presentan una geometría complicada y resultan útiles para conocer las deformaciones experimentadas a lo largo de la vida útil de las mismas.

La durabilidad de la estructura ha sido satisfactoria, presentando únicamente una pequeña oxidación, debido al escaso recubrimiento que tenían algunas armaduras en la cara exterior, dada la dificultad del ferrallado y hormigonado de esas zonas. La evaluación estructural ha mostrado una seguridad adecuada utilizando los métodos actuales recogidos en la EHE-08, por lo que la estructura puede mantenerse en servicio. Las intervenciones de mantenimiento deben enfocarse en la limpieza de la lámina, protección de la armadura descubierta, relleno de coqueras, aplicación de una capa de protección exterior frente a las agresiones del ambiente y, en su caso, al repintado de la superficie interior de la misma para que recupere el aspecto estético original.

\section{AGRADECIMIENTOS}

Este trabajo se engloba en los proyectos intramurales 201060 E118 «Investigación de hormigones especiales avanzados para aplicaciones estructurales en ingeniería y arquitectura, eficientes desde el punto de vista técnico, económico y medioambiental» y 201360E016 «Investigación del deterioro de los sistemas constructivos en edificios del patrimonio construido. Técnicas avanzadas de inspección, diagnóstico y rehabilitación sostenible», financiados por el CSIC, del Ministerio de Economía y Competitividad. Asimismo, los autores quieren agradecer a la empresa Grafinta S.A. su ayuda en la toma de los datos geométricos de la lámina. 


\section{REFERENCIAS}

(1) IETcc. (1999). Informes de la Construcción. Costillares es así (Especial centenario Eduardo Torroja), 51(462), doi: http://dx.doi.org/10.3989/ic.1999.v51.i462.

(2) Azorín, V., Cassinello, P., Monjo, J. (2012). Archivo Eduardo Torroja. La Sede del itcc (1949-1953). Inéditos anteproyectos previos a su construcción. Informes de la Construcción, 65(525): 5-18, doi: http://dx.doi.org/10.3989/ic.11.023.

(3) Cassinello, F., Torroja, J.A., Morán, F., Fernández, R. (1969). Morfogénesis de una lámina. Informes de la Construcción, 22(214): 3-26.

(4) Presidencia del Gobierno. (1968, 3 de diciembre). Decreto 2987/1968, de 20 de septiembre, por el que se aprueba la instrucción para el proyecto y la ejecución de obras de hormigón en masa o armado. Boletín Oficial del Estado, $\mathrm{n}^{0} 290$ : 17257-17291. España.

(5) Arroyo, J.L., Morán, F., García-Meseguer, A. (2009). Jiménez Montoya. Hormigón Armado. Barcelona: Editorial Gustavo Gili.

(6) Presidencia del Gobierno. (2008, 22 de agosto). Real Decreto 1247/2008, de 18 de julio, por el que se aprueba la instrucción de hormigón estructural (EHE-08). Boletín Oficial del Estado, n⿳0 203: 35176-35178 y Suplemento.

(7) Echevarría, L., Garnica, C., Gómez, R., Gutiérrez, J.P. (2014, 1-4 de abril). Estudio del comportamiento de una lámina de hormigón armado situada en el patio de Alarifes del Instituto de Ciencias de la Construcción Eduardo Torroja. En Actas del Congreso Latinoamericano sobre Patología de la Construcción, Tecnología de la Rehabilitación y Gestión del Patrimonio REHABEND 2014. Santander: Universidad de Cantabria. 